In the symposium as a whole, the present writer got the impression that many zoologists preferred the more robust factual papers, and regretted the necessity for mathematical theory-useful as it has been in the more delicate and abstract sciences of physies and chemistry.

Michael Graham

\section{INBREEDING AND HYBRID VIGOUR IN CROPS AND LIVESTOCK}

$\mathrm{A}$

$T$ the British Association meeting in Brighton, Sections M (Agriculture), K (Botany) and D (Zoology) held a joint discussion on September 10 to discuss "Inbreeding and Hybrid Vigour in Livestock and Crop Improvement". The chair was taken by Sir John Russell.

The symposium was opened by Dr. J. M. Rasmusson, director of the Swedish Sugar Company's Breeding Institute, at Hilleshög, Sweden, with an account of his extensive work on the improvement of Swedish sugar beet. The chief effects of inbreeding sugar beet are to inerease genetical homogeneity and to decrease productivity. At the same time, the adaptability of the plants to varying environmental conditions is lowered while the effects of environmental differences are increased : the plants are, in fact, less able to tolerate and adjust themselves to changes in extermal circumstances. The chemical constitution of the root, an important consideration in sugar beet, is scarcely affected by inbreeding.

The chief value of inbreeding is therefore to produce lines with specific and reliable features. It may also be of use in isolating lines in which troublesome associations between desirable and undesirable char. acters have been broken by changing the linkage relations of the genes. No practically useful types have been produced by continued self-pollination in Sweden; but a less-rigorous type of inbreeding, based on the use of maternal progenies between which selection is exercised, has given valuable lines. It has been observed, too, that rigorous mass selection, especially where the number of parent plants is kept small in each generation, produces the practically useful results of loose inbreeding.

When these various partially inbred lines have been raised, suitable mixtures of them can be used in bulk crosses. When this is done, nearly all the seed is from crossing though, of course, all the $\frac{n(n-1)}{2}$ crosses possible between the $n$ lines are present more or less equally in the bulk. The bulk crosses are of commercial value because of their high yields and adaptability to environmental circumstances. The more different in origin were the parent lines, the greater the improvement on crossing; and crosses between lines produced by mass selection under different conditions generally excel those between lines raised by selection between maternal progenies. The effect of crossing, like that of inbreeding, on the chemical constitution is small. Sugar percentage may be slightly reduced though not to an extent sufficient to offset the increase in root yield.

Dr. Rasmusson concluded by mentioning a special case of crossing. Tetraploids, produced by colchicine treatment, yield 5-10 per cent less sugar than the comparable diploids. But the triploids from crossing diploid and tetraploid exceed the diploids by some 5 per cent. The triploid lines cannot, of course, be maintained from seed; but bulk crossing of diploids and tetraploids yields a mixture of the three chromosome types the average productivity of which sufficiently exceeds that of the diploid to make it commercially valuable.

Prof. R. A. Fisher next dealt with the genetical theory of inbreeding, which, as he pointed out, is essentially a means of manipulating the genetic materials so as to control their strueture and composition. Inbreeding produces lines of predictable behaviour, particularly in their properties in subsequent crosses, even in respect of the numerous genes the effects of which are so small as to preclude their being followed by the mendelian method. Inbreeding is a tool the success of which has been so great in plant improvement that it can scarcely fail to achieve wide use in both plant and animal improvement in the future. Yet a theoretical understanding of its operation seems largely to be lacking, and very diverse opinions are held at present as to the reason for its success.

It is clear that the effect of inbreeding, particularly the production of an inbreeding depression which can be wiped out by one generation's crossing, must imply dominance of the genes concerned, and the genetical theory of the origin of dominance throws some light on how this could come about. The lines isolated by inbreeding differ greatly in their genetical qualities, and selection ean be exercised among them at three stages in their production, namely, in the choice of individuals with which to start the inbreeding, in the choice of individuals for continuing the lines during the inbreeding, and in the choice of lines, once established as true-breeding, for use, especially in subsequent crosses. Of these, selection among the established lines is the most valuable, because the true-breeding nature of these lines permits selection based on extensive testing of a wide variety of them. Indeed, this facility offered for increasingly accurate selection is the essential advantage conferred by inbreeding. The rapid improvement in the accuracy of variety trials, made possible by replicated and randomized field designs, has played an important part in refining the selection which has been so successfully practised in the production of hybrid maize.

Mr. G. M. L. Haskell, who followed Prof. Fisher, gave an account of the theory and practice of hybrid maize production. Hybrid maize represents so great an advance over the open-bred varieties which preceded it that it has now replaced the latter virtually completely in the corn belt of the United States. Maize is naturally a cross-pollinator, and genetically the plants are mostly heterozygous. Ordinary methods of selection had shown themselves incapable of materially improving the open-bred varieties of maize before the new methods were introduced. The introduction of the inbreeding technique has led to the production of a great variety of lines which, though all showing inbreeding depression in greater or lesser degree, are uniform and virtually truebreeding and have satisfactory plant characters. Hybridization of inbreds, which is made easy by the monœcious habit of the plants, produces offspring the hybrid vigour of which is often such that they surpass in yield not merely the inbreds themselves but also the open-bred varieties from which these inbreds were produced. Every one of the hybrid plants is a potential yielder, and the hybrid families are uniform 
in appearance, time of maturity and disease resistance. The more diverse the parentage of the inbreds, the more likely they are to combine well in giving high. yielding hybrids. Combining properties vary from inbred to inbred and represent a character for which breeding can deliberately be practised.

Mr. Haskell described the commercial methods used in the United States for inbreeding and hybridizing maize, both in sweet corn where first crosses are used for commercial production, and in field corn where double crosses, made between two first crosses, are preferred. The special problems of breeding maize suitable for Great Britain in regard to photoperiodism and cold-hardiness were mentioned in conclusion. A feature of Mr. Haskell's paper was the wealth of lantern slides with which all its points were illustrated.

In the fourth contribution to the discussion Dr. H. P. Donald dealt with the use of inbreeding and crossbreeding in livestock production. Breed crossing is not an uncommon practice in animal husbandry. Sometimes it is indiscriminate, particularly where, as in Britain, many breeds are kept. Such crossing is not intrinsically undesirable though it must always indicate a lack of purpose in the breeding aims. Other crosses are made deliberately for purposes such as that of distinguishing the sexes at hatching in poultry. Crossbred animals may also be better fitted to particular environments. For example, the Zebu-cattle crosses in northern Australia combine good heat tolerance and quick growth; but have lost some popularity because of their intractability in handling. In the extreme case breeds might be kept as inbreds solely for the purpose of producing crossbreds. A further type is cyclical crossbreeding, where successive generations are mated to two, three or four breeds in turn. This should conserve the greatest part of any heterosis that the crossbreeding could give without the necessity of always going back to the pure breeds for crossing; but it has yet to be shown that the heterosis so obtained is worth while.

Inbreeding has not so far been widely tested as a preliminary to crossbreeding in livestock, because of the great experimental expense. It is known, however, to result in depression of such characters as viability, fertility and milk production. Many defects like scrotal hernia and cleft palate also appear. Slow inbreeding under selection might help to overcome these troubles, but many of the lines must in any event be discarded. The exploitation of inbreeding followed by erossbreeding is too expensive for the ordinary farmer and must require a new breeding organisation. This has been developed for the 'Hyline' poultry in the United States. These birds resemble double-cross maize in their method of production and they cannot be successfully bred on by the farmer. They show hybrid vigour more in the rate of survival than in the egg production of surviving hens.

The complementary use of inbreeding and crossbreeding in animals has potentialities in three ways: to exploit hybrid vigour, to fit the requirements of new territories, and to improve breeds within their own limits when simple selection ceases to be effective. It remains to be demonstrated, however, in each case whether the gain in production will pay for the labour and expense of the method.

The final paper of the meeting was given by Dr. K. Mather, who discussed the genetical prineiples and problems of inbreeding and crossbreeding as a method of plant and animal improvement. In inbreeding plants, such as wheat, peas and tomatoes, homozygous lines of uniform excellence can be maintained by the natural breeding system of the crop. This is not possible with crossbreeders, in which varieties and breeds, though in equilibrium from generation to generation, must always consist of a heterogeneous collection of heterozygous indi. viduals. Genetical variation is inevitable and the average of the variety must in consequence always be below the best. The homozygotes produced by inbreeding are always poor in such species. Uniform and predictable groups of heterozygotes can, however, be obtained by artificially crossing these homozygous lines. Thus it is the aim of the method of inbreeding and crossbreeding deliberately to pro. duce heterozygotes uniformly approaching the best individuals of the open-bred varieties and so, as groups, excelling the average of the varieties; and further to produce these heterozygotes consistently generation after generation.

Five problems arise with this method of breeding. First, there is the great multiplicity of inbred lines which can be raised and must be tested. In the past the desirable inbreds have been discovered by trial and error. Those which give the best hybrids are not, in fact, always themselves the most productive inbreds. Combining ability is the critical character of the inbreds, and it is on this character that they must mainly be selected. Quicker methods are needed for sorting out the lines with valuable com. bining properties from the mass of their less useful fellows.

The second problem is raised by the poverty of the inbreds themselves. They are expensive to keep, and their immediate yield of hybrid offspring when crossed is low, so that hybrid production is costly. Various methods, such as double-crossing and topcrossing, are used to overcome this latter difficulty without sacrificing the chief benefits of the system of breeding.

The method of convergent improvement has also shown itself capable of raising the vigour and productivity of the inbreds themselves.

Thirdly, there is the problem of making the necessary controlled crosses. This is easy in dicecious animals and in moncecious maize; but plants with hermaphrodite flowers require special methods. Cytoplasmically controlled male-sterility has been used for this purpose in onions, and bulk crossing is being exploited in sugar beet.

The fourth problem is raised by the very uniformity of the inbreds and their hybrids. Where only inbreds are maintained there is a risk of loss of the valuable reservoir of genetical variation which is to be found in the open-bred varieties, and in maize special collections of the old types have become necessary to provide sources of future breeding material. In the hybrids the danger of complete genetical uniformity is that it may result in total loss of the crop through a short period of adverse weather, the effect of which on a more variable stand would be much lese serious. The production of double-cross hybrids goes some way to overcome this difficulty.

Finally, there is the question of whether the raising and crossing of inbreds pays for itself. In maize and sugar beet it certainly does; but elsewhere the answer is less clear. It seems, in fact, that the answer will not be the same for all species in all circumstances, and any investigation of the practical possibilities of inbreeding and crossbreeding must therefore pay due attention to the economic aspect.

K. MATHFR 